\title{
Quantifying Brain Connectivity: A Comparative Tractography Study
}

\author{
Ting-Shuo Yo ${ }^{1}$, Alfred Anwander ${ }^{1}$, Maxime Descoteaux ${ }^{2}$, Pierre Fillard ${ }^{2}$, \\ Cyril Poupon ${ }^{2}$, and T.R. Knösche ${ }^{1}$ \\ 1 Max Planck Institute for Human Cognitive and Brain Sciences, Leipzig, Germany \\ ${ }^{2}$ Neurospin / CEA Saclay, Gif-sur-Yvette, France
}

\begin{abstract}
In this paper, we compare a representative selection of current state-of-the-art algorithms in diffusion-weighted magnetic resonance imaging (dwMRI) tractography, and propose a novel way to quantitatively define the connectivity between brain regions. As criterion for the comparison, we quantify the connectivity computed with the different methods. We provide initial results using diffusion tensor, spherical deconvolution, ball-and-stick model, and persistent angular structure (PAS) along with deterministic and probabilistic tractography algorithms on a human DWI dataset. The connectivity is presented for a representative selection of regions in the brain in matrices and connectograms.Our results show that fiber crossing models are able to reveal connections between more brain areas than the simple tensor model. Probabilistic approaches show in average more connected regions but lower connectivity values than deterministic methods.
\end{abstract}

\section{Introduction}

Diffusion-weighted magnetic resonance imaging (dwMRI) provides a noninvasive way to gain insight into the fibre architecture of the brain white matter, and thereby opens a window for the in vivo exploration of the anatomy of neural networks. In the past few years, a number of algorithmic approaches to the reconstruction of nerve fibre tracts from dwMRI have been proposed, collectively known as tractography. However, only few attempts have been made so far to quantitatively compare these different methods [1]. In this study, we compare a representative selection of state-of-the-art tractography algorithms, using connectivity matrices and connectograms based on a novel quantitative connectivity measure.

Most of the current techniques in dwMRI tractography can be divided into two major components: local modeling of the diffusion propagator or the fibre orientation structure in each voxel, and fibre tracking algorithms integrating this local information into streamlines representing fibre tracts.

Local modelling techniques convert the diffusion weighted MR signal into some quantity that can be used to determine the local fibre directions. There are two major classes of algorithms. The first one comprises methods aiming at a more or less simplified reconstruction of the diffusion propagator. Under the assumption of Gaussian anisotropic diffusion, this leads to the diffusion tensor (DT) model 
[2], which can represent only one main direction within each voxel and therefore fails to capture crossing or branchings of fibre populations. More complex models use e.g. compositions of ellipsoids or cylinders, like the multiple-tensor model 3 and the ball-and-stick model [4. Another type of methods provides a less parameterized representation of the diffusion propagator. For example, if the q-space is completely sampled, one may use the spatial Fourier transform to reconstruct a restricted and blurred version of the diffusion propagator. This method is referred to as q-space imaging (QSI) or diffusion spectrum imaging (DSI) [5]. If only one b-value was used, one may compute the radial integral of the diffusion propagator (q-ball imaging, QBI, 6]) or its persistent angular structure (PAS, [7]).

The second class of methods directly aims at the reconstruction of the distribution of fibre orientations, e.g., by spherical deconvolution (SD) 8]. This approach requires an explicit model of the diffusion properties of a single fibre (convolution kernel). Its results are naturally more directly interpretable in terms of quantitative connectivity measures, as compared to methods that describe the diffusion propagator.

A detailed review of these methods can be found in [9]. In our comparison, we only include such local modelling methods that are suitable for high-angular resolution diffusion imaging (HARDI) data with a single b-value of $b=1000$. This naturally excludes QSI, which would require a complete Cartesian sampling of the q-space. Also QBI, which requires a higher b-value to provide a better angular discrimination than the tensor model [6], is not suitable for our dataset. The selection comprises the DT, multiple ball-and-stick, PAS and SD approaches. It can be considered representative, because it exemplifies all major approaches to the problem: (1) models assuming only one main fibre direction (DT), (2) models that allow for a small number of main fibre directions that has to be determined by some model selection procedure (multiple ball-and-stick), (3) models that represent the angular structure of the diffusion propagator (PAS), and (4) approaches that model the fibre orientation density directly, rather than the diffusion propagator (SD). Most other methods can be assigned to one of these classes (except QSI).

Based on these local models, tractography techniques integrate the local information connecting the voxels. There are two major approaches. With deterministic tractography, the reconstructed fibres are exclusively guided by the most likely directions in each voxel. In contrast, probabilistic fibre tracking methods repeat the streamlining process multiple times, each time with a new set of directions drawn from a probability distribution, which is based on the local model. In this study we evaluate each local model with both of these approaches, except for the multiple ball-and-stick model, which is only used with probabilistic tractography. The resulting collection of techniques also covers a range of software packages, which have been used in a large number of studies (see table 1).

For the quantitative comparison of the methods we focused on a type of information that is most naturally associated with tractography, namely if, and to what degree, two regions in the brain are connected by nerve fibres. This measure might also be a useful way to express prior information on connectivity 
within various techniques for modelling functional networks in the brain, such as dynamic causal modelling [10.

The remainder of the paper is organized as follows. In section 2 , the evaluated dwMRI tractography algorithms and the operational definition of the quantitative connectivity is presented in detail. The results of the experiments are shown in section 3, and details of difference among methods are discussed in section 4 .

\section{Methods}

Dataset and Regions of Interests. All compared methods are applied to the HARDI dataset of one human subject. Diffusion images were acquired on a Siemens 3T Trio scanner with isotropic resolution of $1.7 \mathrm{~mm}$ (60 directions, $\mathrm{b}=1000 \mathrm{~s} / \mathrm{mm} 2$, GRAPPA/2, NEX3). Data is corrected for subject motion and registered to the anatomical $\mathrm{T} 1$ weighted image.

Fourteen language-related brain regions are selected as the regions of interests (ROI) for the quantification of anatomical connectivity (see Fig. 1). Eleven of the selected areas are located on the cortical sheet. In these cases the ROIs are placed at the interface between white and grey matter, which is defined as those voxels with fractional anisotropy (FA) greater than 0.15 , which neighbour voxels with an FA of less than 0.15 . Three additional regions comprise a mid-sagittal cross section of the corpus callosum, a horizontal cross section of the pyramidal tract, and the surface of the thalamus. The size of each selected ROI can also be found in figure 1

Compared Algorithms. Table 1 summarizes all algorithms and software packages used for fibre reconstruction. The concept and implementation of each algorithm can be found in the corresponding references.

Definition of Quantitative Anatomical Connectivity. We define a measure, which reflects the influence the mean neuronal activity in one region has

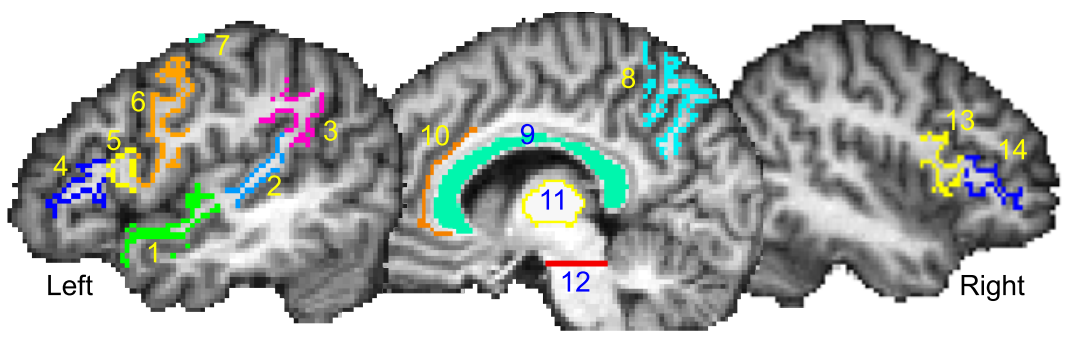

Fig. 1. Locations of selected regions of interests (ROIs). The names and sizes (number of voxels) are: 1. anterior superior temporal gyrus (STG) (497); 2. posterior STG (378); 3. angular gyrus (507); 4. Brodmann area 45 (BA 45) (319); 5. BA 44 (164); 6. precentral gyrus (PCG) ventral (796); 7. PCG dorsol (615); 8. precuneus (731); 9. corpus callosum $(316) ; 10$. anterior cingulate $(155) ; 11$. thalamus $(385) ; 12$. cortical spinal tract $(180) ; 13$. BA45, right hemisphere $(400) ; 14$. BA44, right hemisphere (347). 
Table 1. DWI tractography methods included in the comparison and related references. The computation time is mainly dominated by the local model fitting, and can be different due to the implementation.

\begin{tabular}{|c|c|c|c|}
\hline Local Model & $\begin{array}{l}\text { Comp. } \\
\text { Time }\end{array}$ & $\begin{array}{l}\text { Probabilistic } \\
\text { Tractography }\end{array}$ & $\begin{array}{l}\text { Deterministic } \\
\text { Tractography }\end{array}$ \\
\hline Diffusion tensor (DT [2]) & $\sim 10 \mathrm{sec}$ & 11 & MedINRIA $^{1} 12$ \\
\hline Multiple ball-and-stick 4 & $\sim 2$ days & $\mathrm{FSL}^{2}$ & - \\
\hline Sph. deconv. (SD[8]) & 20-120 min. & $\operatorname{MRtrix}^{3}$ 8] & BrainVisa $^{4} 13$ \\
\hline Per. ang. str. (PAS[7]) & $\sim 1$ month & Camino $^{5} 14$ & Camino 15 \\
\hline
\end{tabular}

on the mean activity in another region. If $W_{A}$ and $W_{B}$ are the sizes of the start and target regions, respectively (proportional to the number of output neurons as well as to the number of voxels), and $F$ is the number of fibres connecting the two regions (proportional to the number of tracts, random walks, or similar), then the influence $C_{A \rightarrow B}$ of the mean activity of the start region $N_{A}$ onto the mean activity of the target region $N_{B}$ can be derived as follows. The mean activity $N_{B}$ can be computed as the cumulative activity on the fibre tract $N_{F}$ divided by the size of the target region $W_{B}$. The cumulative activity $N_{F}$ is in turn proportional to the product of the mean activity of the start region $N_{A}$ and the number of fibres $F$. The connectivity can then be computed as:

$$
C_{A \rightarrow B}=\frac{N_{B}}{N_{A}} \propto \frac{F}{W_{B}},
$$

This connectivity measure is used throughout the comparison.

For probabilistic algorithms, we simulate fibres from each source point (e.g. voxel) $n$ times. The connectivity is then computed as the ratio of the fibres that reach the target region divided by $n$ times the number of source points in region $B$. For deterministic algorithms, we generate fibres starting from each voxel with $\mathrm{FA}>0.15$ in the entire brain. We then count the number of fibres that run through $A$ and $B$, as well as the number of fibres that run through $A$. The ratio of these two numbers multiplied by the ratio of the regions' sizes is then taken as an estimate of the connectivity.

\section{Results}

Figure 2 shows the logarithm of derived connectivity measures for each method in the matrix form. The ROIs are sorted with the spectral reordering algorithm [16] so that ROIs with high connectivity values will be clustered together. To

\footnotetext{
${ }^{1}$ http://www-sop.inria.fr/asclepios/software/MedINRIA/

2 http://www.fmrib.ox.ac.uk/fsl/

3 http://www.nitrc.org/projects/mrtrix/

4 http://brainvisa.info/

5 http://www.cs.ucl.ac.uk/research/medic/camino/
} 
avoid confusion, all matrices are presented in the same ordering, which is based on the connectivity value derived from FSL. By comparing figure 2 with figure 1. we can see that anatomically closer areas are always clustered together, and the disconnection between the left and right hemisphere is very obvious.

Among different algorithms, the pattern of the connectivity matrix are similar, but the magnitude of connectivity values differ. A darkly shaded row of $c c$, which represents a high connectivity toward the corpus callosum, can be found throughout all methods. Also, the general pattern of highly connected regions is consistent across different methods.

The difference between deterministic (right column) and probabilistic (left column) tractography can also be seen in figure2. Both deterministic tracking with DT and SD show significant white areas (i.e., no connection) in the matrices, while their probabilistic counterparts fill up almost the whole matrices. In addition, for SD, PAS and DT with probabilistic tractography, the shaded areas are lighter (i.e., lower connectivity) than those with deterministic fibre tracking algorithms.

Another way to visualize the quantitative connectivities is by graphs, called connectograms. The vertices are placed in the positions which approximately represent the locations of the ROIs, and the edges represent the magnitude of the connectivity measure. Figure 3 shows the resulting connectograms. In the connectograms, connectivity values above $10^{-1}$ are shown in red, $10^{-2}$ are in blue, and $10^{-3}$ in green. Edges with connectivity values bellow $10^{-3}$ are not shown, and the arrows represent the direction of connection. All methods show many arrows pointing toward vertex 9 , corpus callosum, which is consistent with the heavily shaded rows in figure2, The higher connectivity in deterministic tractography and more connections in more complicated local models can also be found in the connectograms. However, the PAS model shows less edges in probabilistic than deterministic tractography due to the in average lower connectivity values.

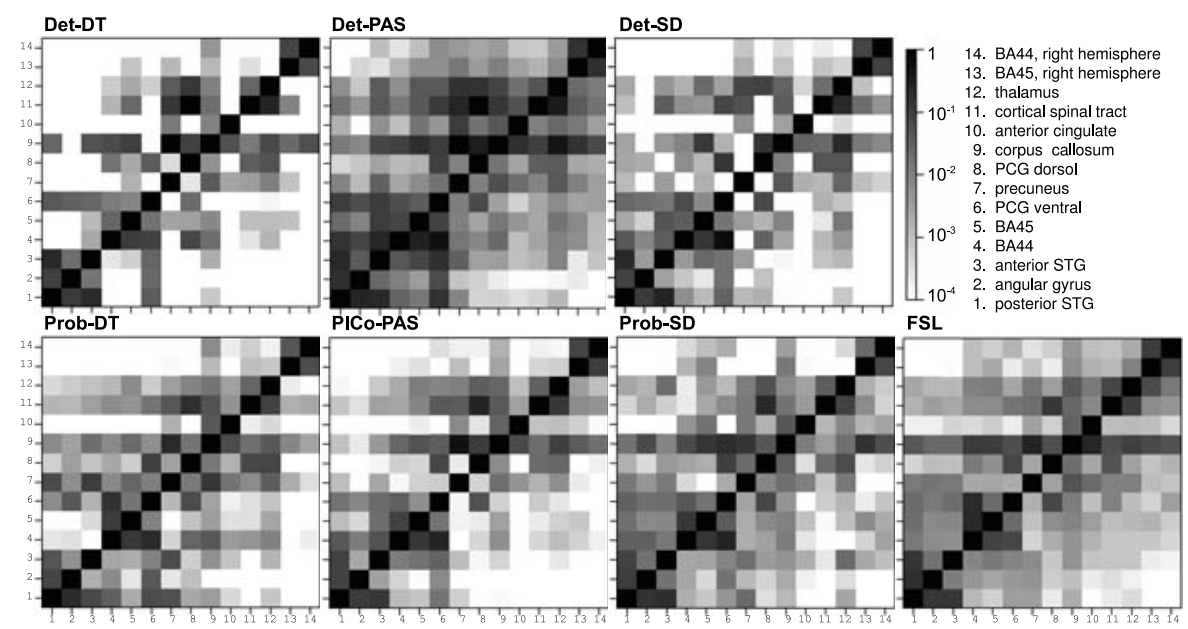

Fig. 2. Connectivity matrices derived from the collection of DWI tractography algorithms in logarithmic scale 

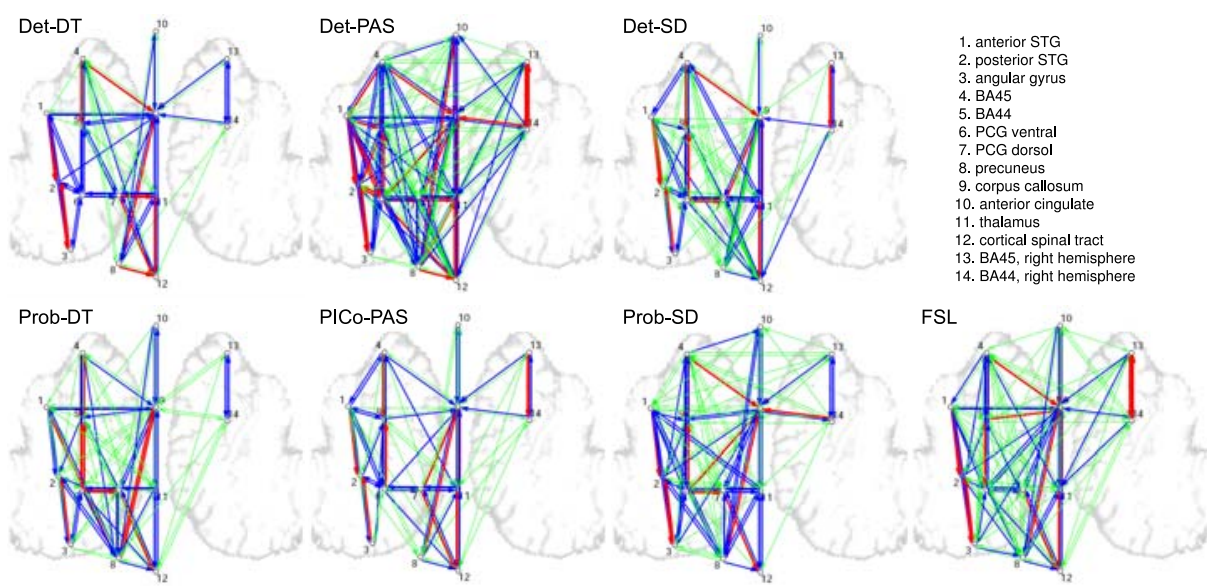

Fig. 3. Connectograms of DWI tractography algorithms. Connectivity values above $10^{-1}$ are shown in red, $10^{-2}$ are in blue, and $10^{-3}$ in green.

Due to the difference in theory and implementation, none of the compared methods give identical results to another. Nevertheless, there is clearly a great degree of similarity. The Mantel test is a technique used to estimate the resemblance between two proximity matrices computed about the same objects. This technique computes a covariant statistic between the two matrices, and then tests it against the null hypothesis of "no association" based on a nonparametric distribution obtained from permuting rows and columns together in one matrix. Several covariant statistics have been designed for different purposes, and we chose the Spearman rank correlation, $\rho_{M}$, as recommended in [17.

The Mantel test based on 1000 random permutations is applied to the connectivity matrices derived from all methods. As expected, all results are significantly correlated ( $p=0$, i.e., none of the 1000 permutations can produce a higher correlation). This result not only further confirms the similarity we observe from the shaded matrices and the connectograms, but also shows the proposed connectivity measure does retain certain structural information which is consistent across dwMRI tractography methods.

\section{Discussion}

Differences Among Methods. Considering the nature of the evaluated methods one would expect two major differences among the methods.

First, deterministic methods as compared to probabilistic ones are expected to feature a sparser connectivity matrix, i.e. there are fewer connections, but with higher connectivity values. This is due to the fact that probabilistic tractography produces a greater variability of fibre trajectories. In deterministic algorithms, fibres tend to follow the same trajectories to a much higher extent, resulting in more extreme connectivity values, i.e. two areas are more likely either strongly connected to not at all. 

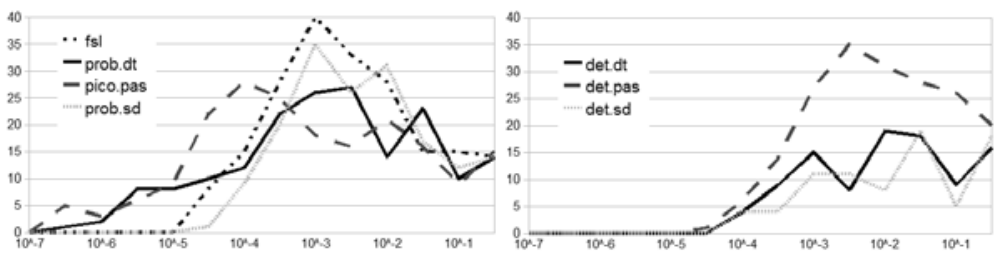

Fig. 4. Histograms of the connectivity values in logarithmic scale (zeroes are not counted). The left panel shows the histogram of the probabilistic tractography methods, and the right panel shows the deterministic ones.

Looking at the results, it turns out that this prediction clearly holds for the DT and SD model, but not for PAS. For PAS, the probabilistic PICo algorithm produces a clearly sparser connectivity matrix and connectogram than deterministic tracking. The reason for this could be the threshold we put at $10^{-4}$ in the matrix and $10^{-3}$ in the connectogram. Figure 4 shows the histograms of compared methods. It is clear that the histogram for PAS with deterministic tracking has a mode in $10^{-3}$, and it with PICo in $10^{-4}$.

The second prediction would be that, with the same tracking method, local models which can represent multiple fibre orientations (e.g. PAS, ball-and-stick, $\mathrm{SD})$ will generate more connections between areas. This is logical because the additional fibre orientations might lead to new fibres that cross the major tracts. From our results, this prediction is only partially confirmed, since this trend is not as strong in the probabilistic tracking.

Concluding Remarks. In this study, we have compared a collection of stateof-the-art dwMRI tractography algorithms based on a quantitative connectivity measure. It has been shown that the proposed criterion give similar patterns across different methods, and also reasonably distinguish algorithms from each other. The results suggest that local models represent multiple fiber orientations can reconstruct more connections with a cost of more computation time (see table 1), as well as the probabilistic tractography. Since the difference in computational cost of tractography algorithms does not differ much, the choice of local models may dominate the computational resource required for this task. Although the optimal combination of methods can not be concluded from our findings, this study proposes a methodology to quatitatively compare different methods, which is of utmost importance for the community.

Future work will be focused on validating the comparison across different subjects, and to find a proper way to incorporate the quantified brain connectivity with other brain modelling techniques.

\section{References}

1. Descoteaux, M., Deriche, R., Knosche, T.R., Anwander, A.: Deterministic and probabilistic tractography based on complex fibre orientation distributions. IEEE Transactions on Medical Imaging 28(2), 269-286 (2009) 
2. Basser, P.J., Mattiello, J., Lebihan, D.: Mr diffusion tensor spectroscopy and imaging. Biophys. J. 66(1), 259-267 (1994)

3. Parker, G.J., Alexander, D.C.: Probabilistic monte carlo based mapping of cerebral connections utilising whole-brain crossing fibre information. In: Taylor, C.J., Noble, J.A. (eds.) IPMI 2003. LNCS, vol. 2732, pp. 684-695. Springer, Heidelberg (2003)

4. Behrens, T.E., Berg, H.J., Jbabdi, S., Rushworth, M.F., Woolrich, M.W.: Probabilistic diffusion tractography with multiple fibre orientations: What can we gain? Neuroimage 34(1), 144-155 (2007)

5. Wedeen: Mapping fiber orientation spectra in cerebral white matter with fourier transform diffusion mri. In: Proc. of the 8th ISMRM, p. 82 (2000)

6. Tuch, D.S.: Q-ball imaging. Magn. Res. in. Med. 52(6), 1358-1372 (2004)

7. Jansons, K.M., Alexander, D.C.: Persistent angular structure: new insights from diffusion magnetic resonance imaging data. Inv. Prob. 19, 1031-1046 (2003)

8. Tournier, J.-D., Calamante, F., Connelly, A.: Robust determination of the fibre orientation distribution in diffusion mri: Non-negativity constrained super-resolved spherical deconvolution. NeuroImage 35(4), 1459-1472 (2007)

9. Alexander, D.: Multiple-fibre reconstruction algorithms for diffusion mri. Ann. N.Y. Acad. Sci. 1064, 113-133 (2005)

10. Friston, K.J., Harrison, L., Penny, W.D.: Dynamic Causal Modelling. NeuroImage 19(4), 1273-1302 (2003)

11. Anwander, A., Tittgemeyer, M., von Cramon, D., Friederici, A., Knosche, T.: Connectivity-based parcellation of broca's area. Cereb. Cor. 17, 816-825 (2007)

12. Toussaint, N., Souplet, J., Fillard, P.: Medinria: Medical image navigation and research tool by inria. In: Proc. of MICCAI 2007 Workshop, Australia (2007)

13. Cointepas, Y., Poupon, C., Maroy, R., Rivire, D., Le Bihan, D., Mangin, J.F.: A freely available anatomist/brainvisa package for analysis of diffusion mr images. In: Proc. 9th HBM CD-Rom Neuroimage, New York, vol. 19(2) (2003)

14. Parker, G.J., Haroon, H.A., Wheeler-Kingshott, C.A.: A framework for a streamline-based probabilistic index of connectivity (pico) using a structural interpretation of mri diffusion measurements. J. Mag. Res. Imag. 18(2), 242-254 (2003)

15. Cook, P.A., Bai, Y., Nedjati-Gilani, S., Seunarine, K.K., Hall, M.G., Parker, G.J., Alexander, D.C.: Camino: Open-source diffusion-mri reconstruction and processing. In: 14th Scientific Meeting of the ISMRM, p. 2759 (2006)

16. Higham, D.: Unravelling small world networks. Journal of Computational and Applied Mathematics 158(1), 61-74 (2003)

17. Schneider, J.W., Borlund, P.: Matrix comparison, part 2: Measuring the resemblance between proximity measures or ordination results by use of the mantel and procrustes statistics. J. Am. Soc.,Inf. Sci. and Technol. 58(11), 1596-1609 (2007) 